\title{
ESTUDO DAS MODIFICAÇÕES GERADAS PELO ENVELHECIMENTO DE MEMBRANAS ANIÔNICAS TROCADORAS DE ÍONS EM UMA SOLUÇÃO SINTÉTICA DE CuHEDP
}

\author{
Juliana Mendonça Silva de Jesus ' \\ Tatiana Scarazzato ' \\ Daniella Cardozo Buzzi ${ }^{2,3}$ \\ Denise Crocce Romano Espinosa
}

\section{Resumo}

O envelhecimento de membranas trocadoras de íons, comumente utilizadas em processos de eletrodiálise, contribui na avaliação e compreensão de modificações suscetíveis ao processo industrial. Nesse estudo, membranas aniônicas de morfologia distinta foram submetidas ao envelhecimento por imersão, em soluções sintéticas, nas concentrações de $1 \%, 5 \%$ e $10 \%$ v/v de um banho de cobre com ácido I hidroxietano-I, I difosfônico (HEDP). A ação do meio foi verificada durante $400 \mathrm{~h}$ de contato via microscopia eletrônica de varredura com espectroscopia por dispersão de energia de raios-X (MEV-EDS) e análise termogravimétrica (TGA). Os resultados obtidos indicam que no período de contato estudado, foram mantidas, a estabilidade dimensional e térmica das membranas, não evidenciando a necessidade de descarte. Fato este que motiva o uso desses materiais em um sistema de eletrodiálise, quando aplicado para o tratamento do banho de cobre e HEDP.

Palavras-chave: Envelhecimento; Membranas trocadoras de íons; HEDP; Eletrodiálise.

\section{STUDY OF MODIFICATIONS GENERATED BY THE AGEING OF ANIONIC MEMBRANES IN A SYNTHETIC SOLUTION OF CUHEDP}

\begin{abstract}
The ageing of ion exchange membranes, commonly used in electrodialysis processes, contributes to the evaluation and understanding of modifications susceptible in an industrial process. In this study, anionic membranes of different morphology were submitted to immersion in synthetic solutions at concentrations of $1 \%, 5 \%$ and $10 \% \mathrm{v} / \mathrm{v}$ of a copper bath with I-hydroxyethane-I, I diphosphonic acid (HEDP). Solutions effect were verified during 400 hours of contact using scanning electron microscopy with X-ray energy dispersion spectroscopy (SEM-EDS) and thermogravimetric analysis (TGA). The results indicated that in the period of contact studied, the dimensional and thermal stability of the membranes were maintained, not evidencing the need for disposal. This fact motivates the use of these materials in an electrodialysis system, when applied to the bath treatment of copper and HEDP.
\end{abstract}

Keywords: Ageing; lon-exchange membranes; HEDP; Electrodialysis.

\section{INTRODUÇÃO}

A eletrodiálise (ED) baseia-se em um processo de separação por membranas, inicialmente aplicada à dessalinização [ [-3]. Dada sua efetiva capacidade de separação e recuperação de insumos, estudos apresentam a ED como alternativa de tratamento de resíduos industriais [4-6].

A implementação e operação de uma planta de eletrodiálise vincula-se à custos referentes ao sistema de controle, aquisição e manutenção das membranas trocadoras de íons (MTI), que assumem cerca de $42 \%$ do custo total [7].

Dada as particularidades de cada processo de separação e do resíduo a ser tratado, a investigação preliminar sobre a ação do meio ao contato com as MTI e sua posterior caracterização, mostra-se como

'Departamento de Engenharia Química, Escola Politécnica de São Paulo, Universidade de São Paulo - USP, São Paulo, SP, Brasil.

E-mail: julianams.silva@gmail.com

${ }^{2}$ Departamento de Pesquisa em Engenharia e Educação Continuada - DEPEC, Fundação GORCEIX, Ouro Preto, MG, Brasil.

${ }^{3}$ REDEMAT, Universidade Federal de Ouro Preto - UFOP, Ouro Preto, MG, Brasil.

2176-1523 (C) 2020 Associação Brasileira de Metalurgia, Materiais e Mineração. Publicado pela ABM. Este é um artigo de acesso aberto distribuído sob os termos da licença Creative Commons CC BY-NC-ND (Attribution-NonCommercial-NoDerivs) - https:// creativecommons.org/licenses/by-nc-nd/4.0\%. 
uma ferramenta à implementação de membranas em escala industrial. $\mathrm{O}$ envelhecimento refere-se à verificação do comportamento de membranas em condições típicas e controladas. Tal procedimento contribui na investigação dos materiais mais propícios à separação de um meio em específico, além de indicar possibilidades de melhoria dos agentes separadores [8-10].

Em meados de 1980, Kneifel e Hattenbach [I I] avaliaram 3 I tipos de MTI, catiônicas e aniônicas, submetidas a temperaturas de $25^{\circ} \mathrm{C}$ a $85^{\circ} \mathrm{C}$ e soluções de $0,05 \mathrm{a} I \mathrm{~mol} \mathrm{~L}-1$ de $\mathrm{NaCl}$, durante cinco anos.

Apesar da variação de metodologias de envelhecimento, a investigação de suas propriedades intrínsecas como permeseletividade, grau de inchamento e a caracterização microestrutural tem sido seguida.

Robinson et al. [12] realizaram a revisão de cerca de 50 artigos que relatam o estudo do envelhecimento de membranas poliméricas. Os autores identificaram que a proposta comum dos estudos é a conexão das alterações observadas durante $o$ envelhecimento com à previsão do comportamento que as membranas apresentariam em um sistema industrial. No entanto, em muito dos casos, tal proposta não foi alcançada, mostrando a necessidade de mais estudos na área.

Motivado pelo extenso trabalho realizado por Scarazzato [13], na qual a autora avaliou a eficiência da ED para recuperação do banho de cobre à base de HEDP. O presente estudo visa a verificação de duas membranas aniônicas, a heterogênea HDX 200, e a membrana homogênea PC 200 $D$ em um efluente sintético proveniente do banho galvânico desenvolvido por Vargas [14]. Além da caracterização microestrutural e térmica, aplicada a fim de identificar as principais modificações decorrentes do contato com as soluções de CuHEDP.

\section{METODOLOGIA}

\section{I Membranas Trocadoras de Íons (MTI)}

Para $\circ$ presente estudo foram selecionadas duas membranas aniônicas, sendo uma heterogênea, HDX 200, fornecida pela industrial Hidrodex e a PC 200 D homogênea, fornecida pela PCA Gmbh. Essa última foi submetida à lavagem prévia com água destilada por $24 \mathrm{~h}$ sob agitação a temperatura ambiente, a fim de remover o recobrimento salino utilizado como proteção ao material, proveniente de seu processo de manufatura. A membrana heterogênea não foi submetida a tal pré-tratamento pois não possui qualquer recobrimento superficial. A Tabela I apresenta em detalhes as principais características das MTI selecionadas.

A partir dos materiais selecionados e tratados foram segregadas amostras em duplicata, de cada tipo de membrana, totalizando 48 amostras com $4 \mathrm{~cm}^{2}$ cada.

Tabela I. Principais características das membranas aniônicas $[13,15]$

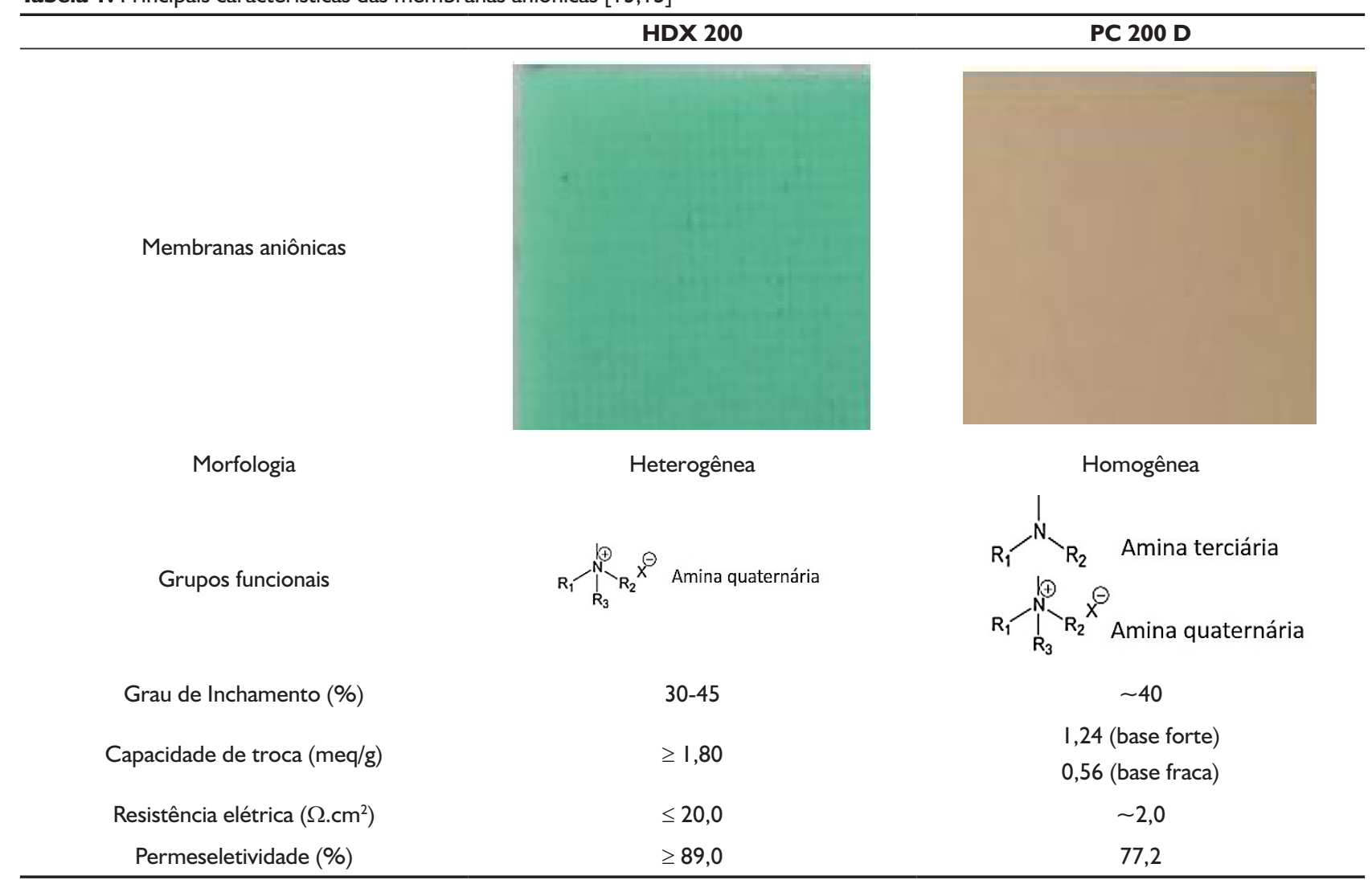




\subsection{Soluções de Envelhecimento}

Para o preparo da solução trabalho utilizou-se reagentes puro para análise, pesados em balança semi-analítica e dissolvidos usando água destilada, conforme descrito na Tabela 2.

O preparo das soluções de envelhecimento deu-se a partir da diluição da solução trabalho nas concentrações I\%, $5 \%$ e $10 \% \mathrm{v} / \mathrm{v}$. Após esse procedimento não foi necessário o ajuste do $\mathrm{pH}$.

\subsection{Caracterização Estrutural MEV-EDS e TGA}

Com a finalidade de se monitorar o efeito do envelhecimento na microestrutura das membranas, amostras virgens e envelhecidas foram avaliadas. Análise por MEV-EDS foi realizada no equipamento de bancada Phenom, modelo Phenom Pro X. Essa técnica foi aplicada a fim de monitorar as possíveis alterações estruturais e a composição da superfície das membranas, com enfoque na detecção de fósforo e cobre.

A análise termogravimétrica foi realizada com o equipamento NETZCSH STA 449, a fim de verificar a estabilidade térmica e a incorporação iônica na membrana por meio da análise do percentual de resíduo final. Os parâmetros de controle para a análise térmica foram: taxa de aquecimento de $20^{\circ} \mathrm{C} \mathrm{min}^{-1}$, atmosfera de nitrogênio e temperatura final de $1000^{\circ} \mathrm{C}$.

Tabela 2. Composição do banho toque de cobre a base de HEDP [13]

\begin{tabular}{cc}
\hline Composição & Concentração \\
\hline ĺons $\mathrm{Cu}^{2+}$ & $4,5 \mathrm{~g} \mathrm{~L}^{-1}$ \\
HEDP & $105,0 \mathrm{~g} \mathrm{~L}^{-1}$ \\
Cloreto de potássio & $7,0 \mathrm{~g} \mathrm{~L}^{-1}$ \\
$\mathrm{pH}$ & $\sim 10,0$ \\
\hline
\end{tabular}

\subsection{Protocolo de Envelhecimento}

O envelhecimento das membranas baseou-se na imersão das amostras, em duplicata; nas soluções de envelhecimento (item 2.2) sob agitação e temperatura ambiente (Figura I). O sistema estabelecido foi mantido fechado com o recobrimento de papel filme para evitar a perda de solução por evaporação durante todo o ensaio.

Dessa forma, as membranas foram mantidas em contato com os meios durante quatro ciclos de envelhecimento: I00h, 200h, 300h e 400h. Ao término de cada ciclo as membranas HDX 200 foram lavadas em água destilada e secas em estufa a $50{ }^{\circ} \mathrm{C}$.

\section{RESULTADOS E DISCUSSÃO}

\section{I Caracterização Microestrutural e Efeito do Envelhecimento}

Para investigar as possíveis modificações que as membranas aniônicas apresentaram decorrente do envelhecimento em soluções de CuHEDP I\%, 5\% e 10\% $\mathrm{v} / \mathrm{v}$, foi verificado, primeiramente, alterações microestruturais e composição superficial das membranas por meio da análise de MEV-EDS. O uso dessa técnica permitiu a avaliação rápida e visual do efeito da interação das membranas e os meios de envelhecimento.

Dessa forma, a Figura 2 apresenta a micrografia das membranas HDX 200 (A) e PC 200 D (B) anteriormente ao processo de envelhecimento. Juntamente a essa figura tem-se a análise por EDS das membranas, a análise realizada foi dada por uma varredura na área total da membrana, e não pontual.

A partir da observação da Figura 2 foi possível verificar a distinção morfológica já esperada entre os materiais estudados. Sendo que a Figura 2A apresenta de forma clara

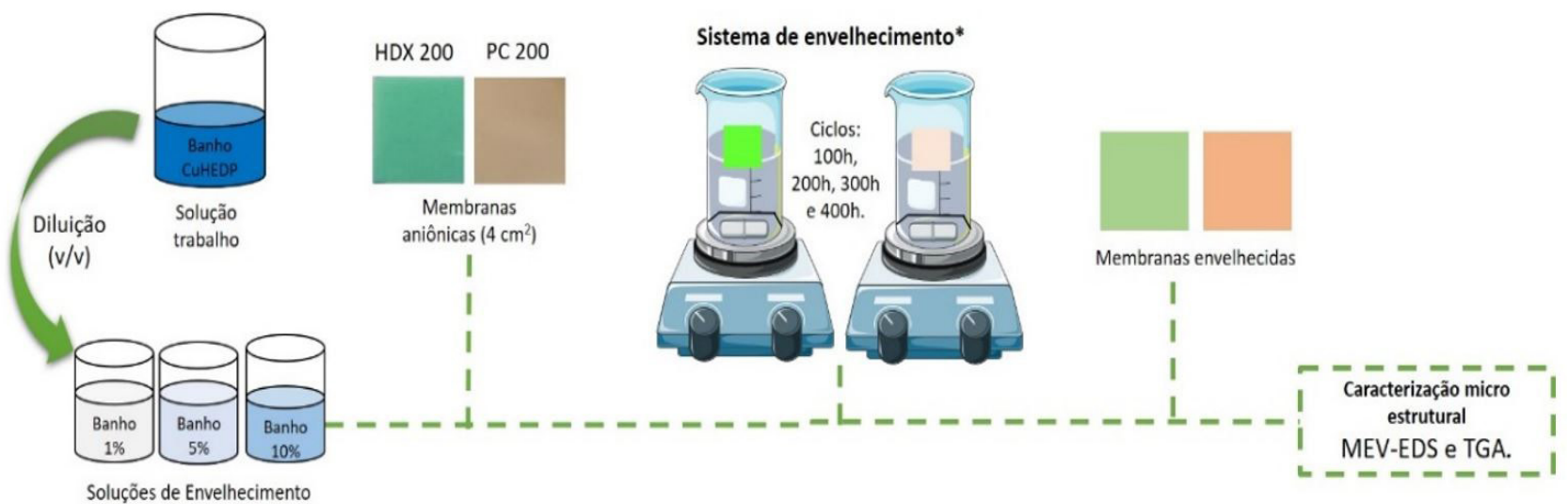

*Realizado em duplicata

Figura I. Representação esquemática do protocolo de envelhecimento das membranas aniônicas PC 200 D (24 amostras) e HDX 200 (24 amostras), ambas com $4 \mathrm{~cm}^{2}$, mantidas em contato com soluções de CuHEDP durante 100h, 200h 300 e $400 \mathrm{~h}$. 
(A)
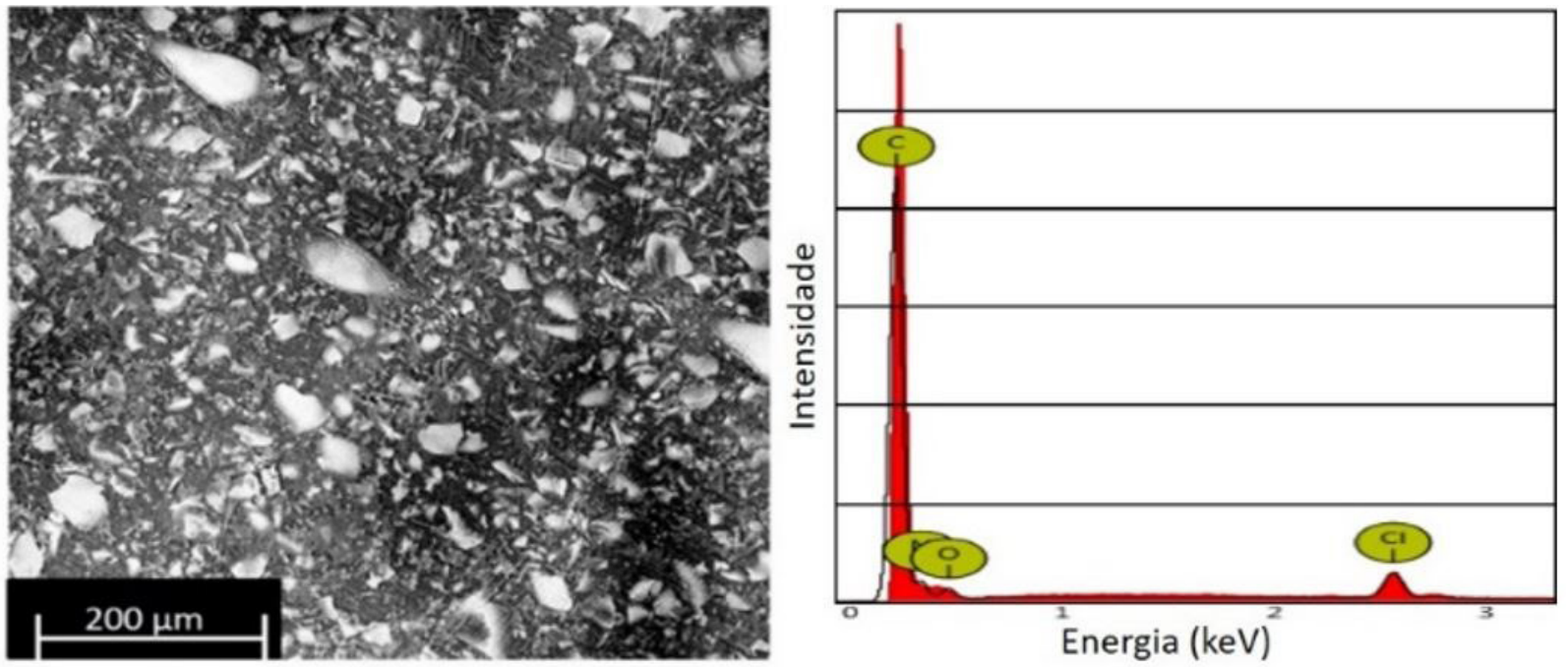

(B)
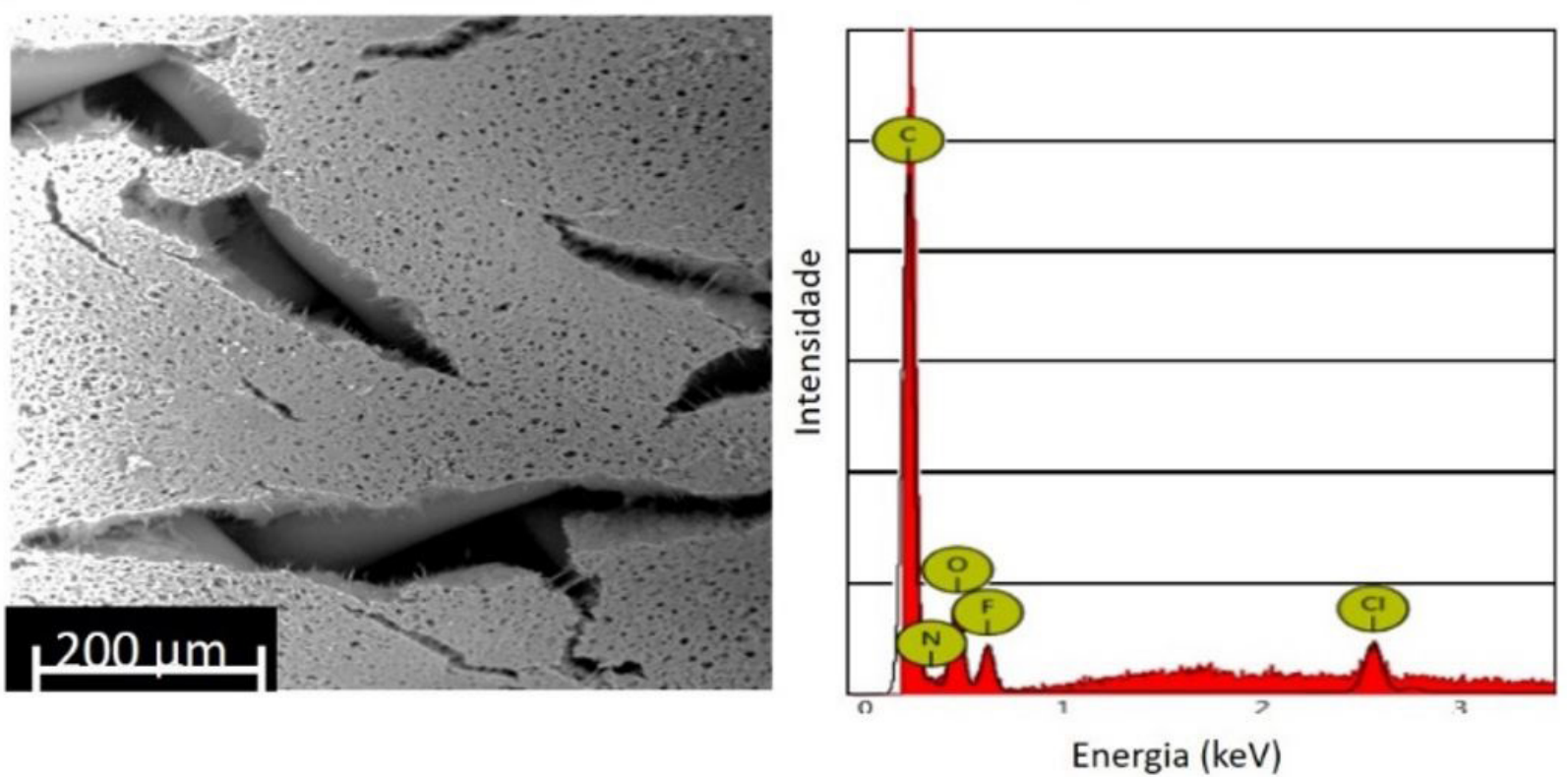

Figura 2. Imagem de elétrons retroespalhados obtida por MEV e espectro de EDS das membranas HDX 200 (A) e PC 200 D (B).

a distribuição da sua composição de forma heterogênea, representada a partir dos pontos brancos posicionados aleatoriamente. Além disso, foi possível verificar que a membrana PC $200 \mathrm{D}$ apresentou em tal condição, hachuras decorrentes do processo de secagem (Figura 2B), ainda que $\circ$ mesmo foi realizado em temperatura ambiente. Comportamento atribuído a sua espessura fina, que é de cerca de 180 a $220 \mu \mathrm{m}$.

Com relação à composição, os espectros EDS de ambos materiais apresentaram sinais similares de carbono, nitrogênio e cloro, diferenciando-se apenas pela presença de flúor na membrana PC $200 \mathrm{D}$, dada pela presença do polímero perfluorado utilizado em sua manufatura. A presença do elemento nitrogênio, em ambas membranas, confirmam a presença dos grupos funcionais, amina terciária e quaternária, tal como observado por Pessoa-Lopes et al. [16].

O efeito médio do envelhecimento sobre as membranas heterogêneas, apresentado na Figura 3, permitiu verificar que em apenas $100 \mathrm{~h}$ foram detectados sinais de incorporação e que a partir de $200 \mathrm{~h}$ sinais de degradação da estrutura polimérica da membrana foram observados. Contudo, não foi possível verificar o efeito das concentrações das soluções de envelhecimento sobre as membranas envelhecidas.

A Figura 4 apresenta 0 resultado referente a HDX 200 envelhecida na solução de CuHEDP I\% v/v durante 400h de ensaio. Tal como obtido pelas imagens de elétrons retroespalhados, os espectros de EDS das membranas envelhecidas apresentaram comportamento análogo entre si. Dessa forma, não foi possível observar o efeito das concentrações das soluções sobre as membranas e nem a distinção do efeito do tempo com relação a composição superficial dos materiais envelhecidos.

Entretanto, o resultado apresentado mostra-se capaz de representar o efeito médio do envelhecimento nas condições estudadas. A Figura 4 apresenta a detecção dos elementos presentes na membrana antes ao processo de 


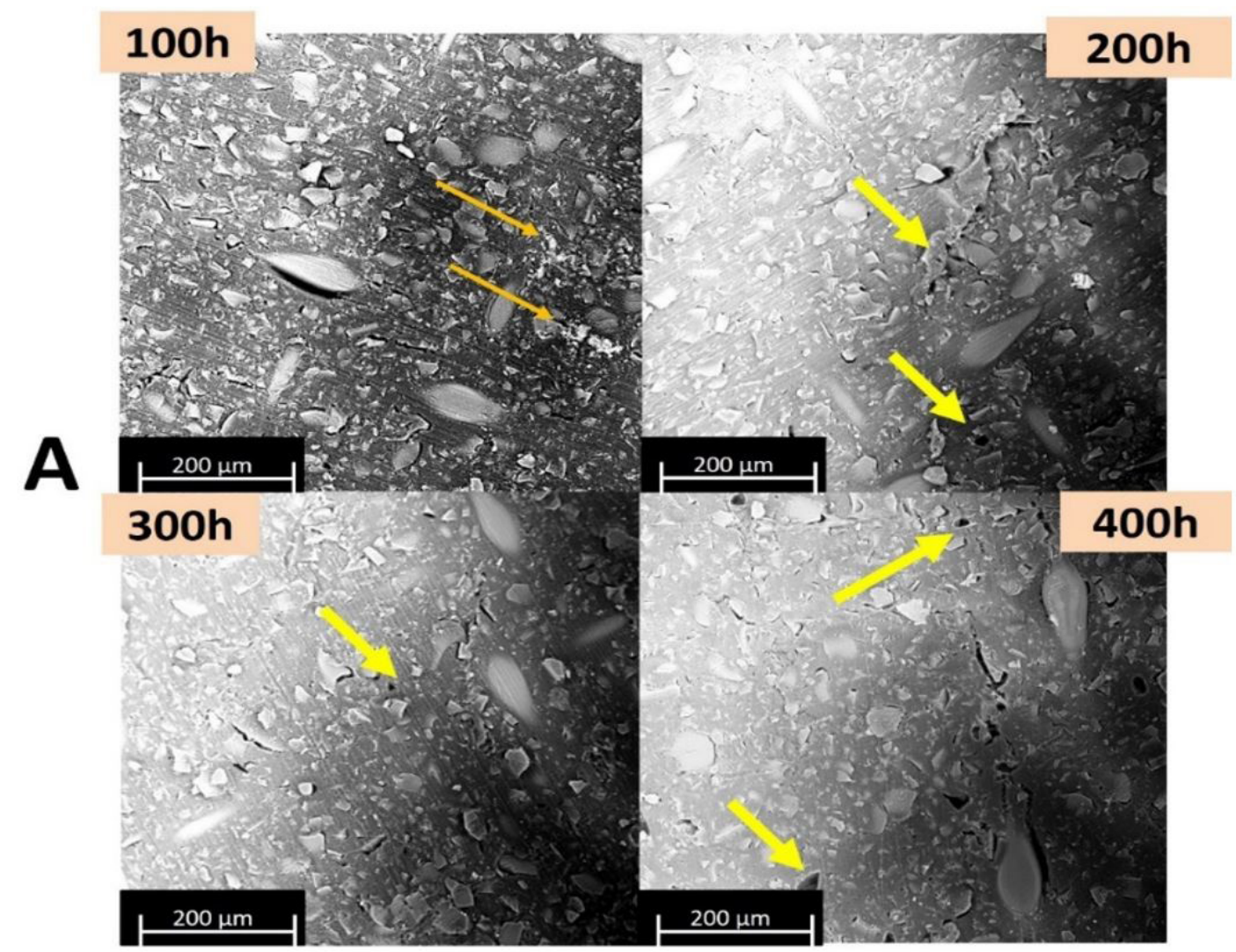

Figura 3. Imagens de elétrons retroespalhados da membrana HDX 200 (A) envelhecidas durante $400 \mathrm{~h}$. As setas laranjas indicam os primeiros sinais de incorporação detectados na membrana HDX 200 envelhecida por 100h em CuHEDP I\% v/v. As setas amarelas indicam os sinais de degradação.

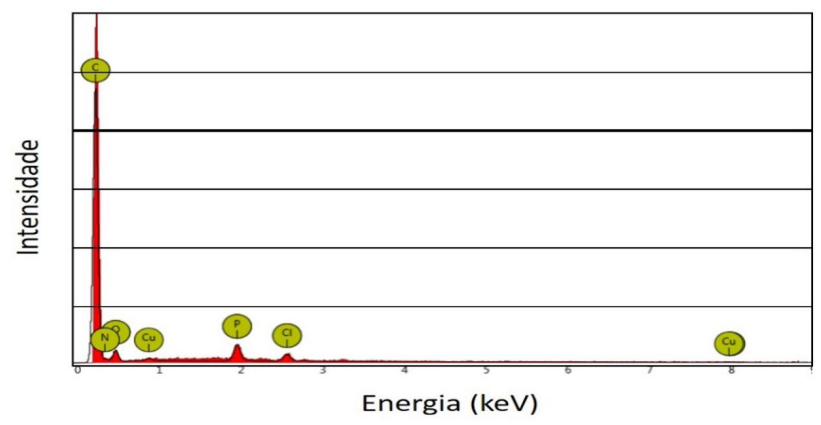

Figura 4. Espectro obtido por EDS das membranas HDX 200 envelhecidas durante $400 \mathrm{~h}$ em CUHEDP I\%v/v.

envelhecimento, além da detecção dos elementos fósforo e cobre, decorrentes da interação membranas-solução. Tal resultado aponta que mesmo após lavagem em água esses materiais apresentaram em sua composição superficial resíduos dos elementos oriundos das soluções de CuHEDP.

Analogamente ao que foi realizado com as membranas HDX 200, após o envelhecimento, as membranas PC 200 D foram submetidas a análise em MEV-EDS (Figura 5). Deve-se destacar que as concentrações dos meios de envelhecimento não manifestaram efeito significativo para os resultados de MEV-EDS, tal como observado nas membranas heterogêneas.
A partir de tais resultados apresentados na Figura 5, foi possível observar que os primeiros indícios de incorporação ocorreram em $100 \mathrm{~h}$ de imersão. No entanto, para os demais ciclos de envelhecimento, $200 \mathrm{~h}$ a $400 \mathrm{~h}$, a interação membrana-solução proporcionou um recobrimento das superfícies das membranas, inibindo a ocorrência das hachuras observadas na membrana inicial. Além disso não foi possível observar pontos de degradação decorrente do processo de envelhecimento.

Assim como observado nas HDX 200 envelhecidas, as análises de EDS para as membranas homogêneas apresentaram resultados similares em composição e intensidade, possibilitando a apresentação de apenas um resultado (Figura 6). Novamente o aparecimento de fósforo e cobre foi atribuído à interação das soluções de CuHEDP às membranas.

\subsection{Avaliação do Efeito do Envelhecimento na Estabilidade Térmica}

Em conjunto com a técnica de imagem utilizada, escolheu-se também o uso da análise termogravimétrica para detectar alterações no comportamento térmico e estrutural das membranas decorrentes do envelhecimento. 


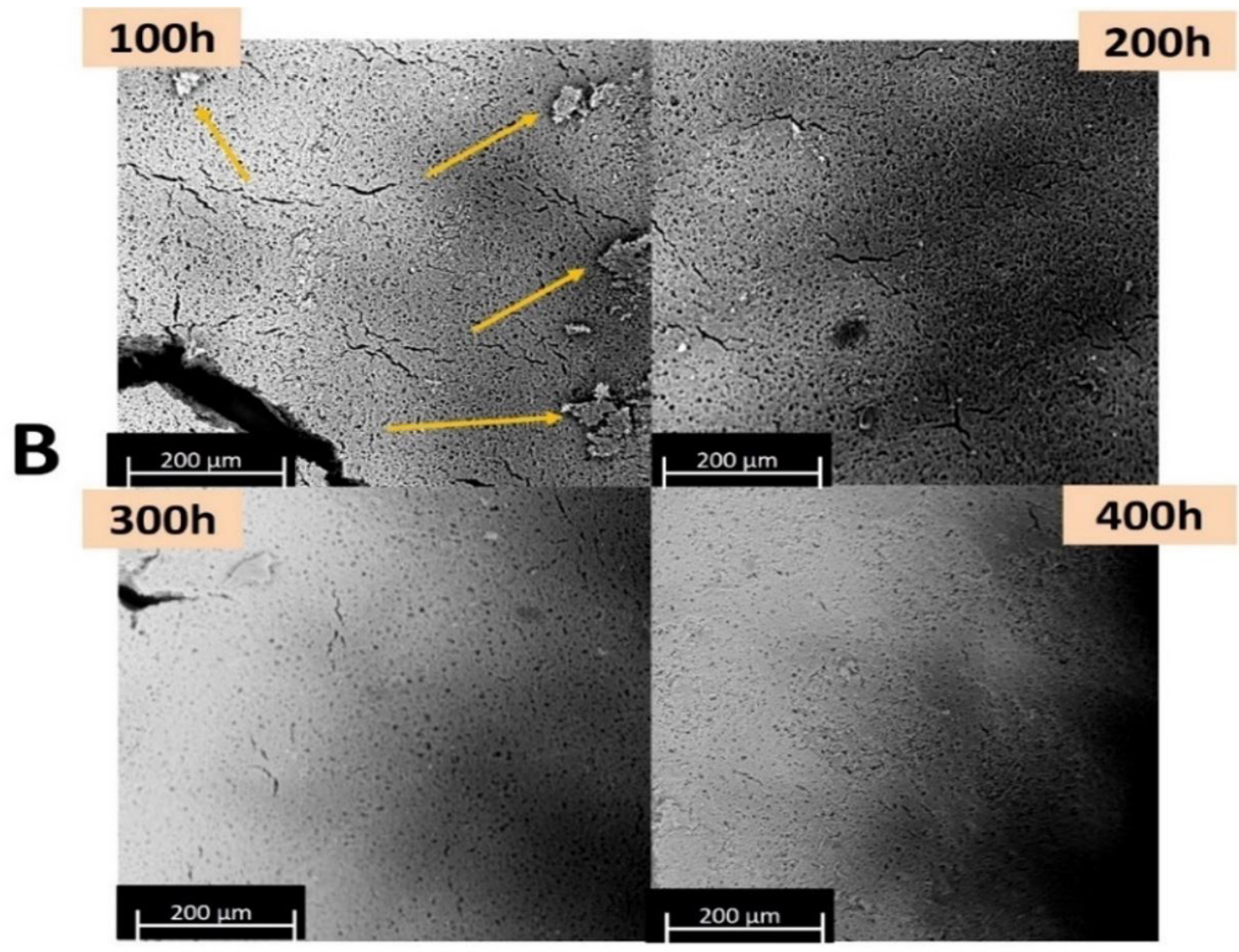

Figura 5. Imagens de elétrons retroespalhados das membranas PC 200 D (B) envelhecidas em 400h. As setas laranjas indicam os primeiros sinais de incorporação detectados na membrana PC 200 D envelhecida por 100h em CuHEDP I\% v/v.

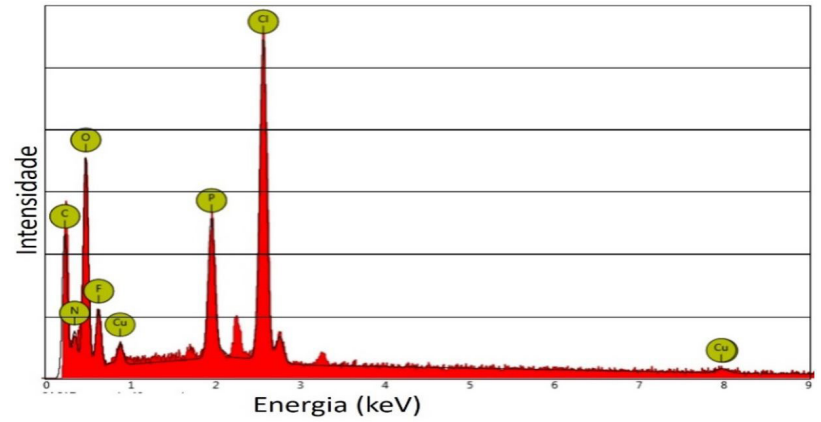

Figura 6. Espectro obtido por EDS das membranas PC $200 \mathrm{D}$ envelhecidas durante $400 \mathrm{~h}$ em CuHEDP I\% v/v.

A Figura 7A apresenta a TGA da membrana HDX 200 e a termogravimetria derivada (DTG) indicando o ponto inicial de cada reação de decomposição do material análise [17]. Pôde-se observar que a decomposição térmica da amostra ocorreu em quatro etapas: a primeira etapa ocorreu em torno de $100^{\circ} \mathrm{C}$, a segunda em aproximadamente $250^{\circ} \mathrm{C}$, a terceira em $480^{\circ} \mathrm{C}$ e a quarta em $600{ }^{\circ} \mathrm{C}$. Segundo a literatura [18-20], a primeira degradação térmica das MTI ocorre entre 60 a $100^{\circ} \mathrm{C}$, que corresponde à desidratação da amostra.
A membrana heterogênea, que possui como grupo funcional a amina quaternária $\left(-\mathrm{NR}_{3}^{+}\right)$, apresentou decomposição em temperaturas entre $250{ }^{\circ} \mathrm{C}$ a $370{ }^{\circ} \mathrm{C}$. Na membrana nova, essa etapa de degradação foi de cerca de $10 \%$ da perda de massa, equivalentemente ao encontrado por Garcia-Vasquez et al. [8]. No entanto, após o envelhecimento as membranas apresentaram modificação térmica com relação a tal etapa, o que pode ser justificado pela incorporação de íons provenientes do meio de envelhecimento (Figura 7B), resultado também identificado pela análise de MEV-EDS.

Além disso, o envelhecimento proporcionou modificações nos percentuais de resíduo final dado pela incorporação iônica na matriz polimérica. $O$ que pôde ser facilmente visualizado pela alteração no perfil de degradação da última etapa da análise termogravimétrica (Figura 7B), etapa essa que era concretizada a partir de $610^{\circ} \mathrm{C}$ nas membranas novas e que foi modificado para $430^{\circ} \mathrm{C}$ após o envelhecimento.

A análise térmica dessas membranas não permitiram avaliar especificamente o efeito das distintas concentrações dos meios de envelhecimento e do tempo de contato, apenas o efeito médio durante $400 \mathrm{~h}$ de ensaio, sendo que os demais períodos de ensaio não apresentaram resultados termogravimétricos significativos e distintos aos apresentados. 


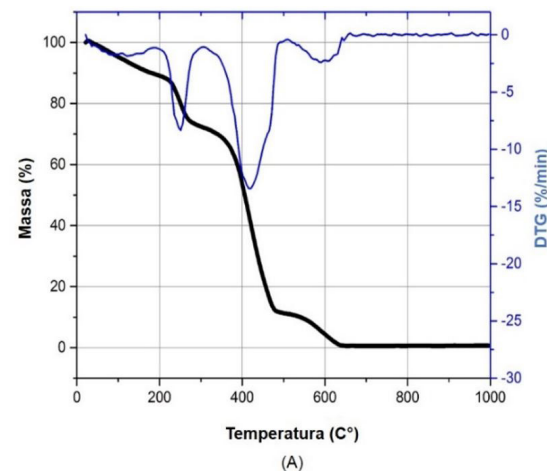

(A)

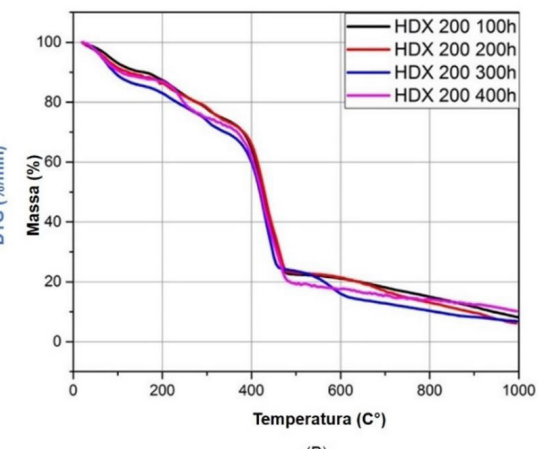

(B)

Figura 7. (A) TGA e DTG da membrana HDX 200; (B) TGA das membranas HDX 200 envelhecidas em soluções de CuHEDP por 400h.

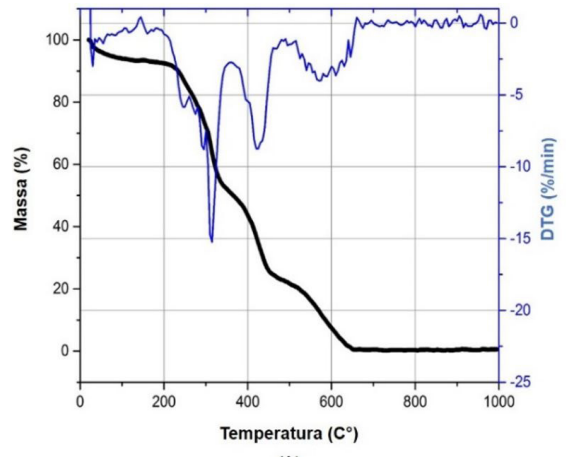

(A)

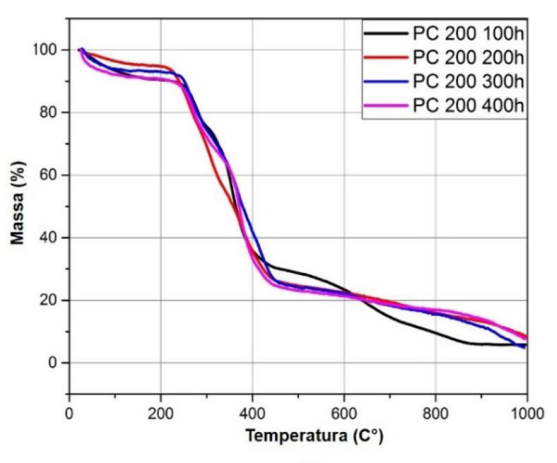

(B)

Figura 8. (A) TGA e DTG da membrana PC 200 D; (B) TGA das membranas PC 200 D envelhecidas em soluções de CuHEDP I\% v/v por 400h.

A Figura 8A apresenta o TGA da membrana PC 200 D, resultado similar ao observado na membrana heterogênea, dada pela decomposição térmica em quatro etapas. A primeira etapa ocorreu a $250{ }^{\circ} \mathrm{C}$, seguida de uma etapa a $300{ }^{\circ} \mathrm{C}$, uma a $400{ }^{\circ} \mathrm{C}$ e, por fim, uma etapa a $650^{\circ} \mathrm{C}$. Conforme anteriormente relatado no item 2. I, a membrana PC 200 D possui dois grupamentos aniônicos. Assim, as duas primeiras etapas foram atribuídas à decomposição dessas ligações aminas. As últimas etapas foram atribuídas à decomposição da matriz polimérica.

A Figura 8B representa o comportamento médio, referente a análise térmica das membranas homogêneas envelhecidas nas distintas concentrações da solução de CuHEDP por $400 \mathrm{~h}$. Equivalentemente ao observado nas membranas heterogêneas envelhecidas, as membranas homogêneas apresentaram modificações na segunda e na quarta etapa (Figura 8B). Além disso, a etapa de degradação da matriz polimérica apresentou efeito do envelhecimento, dada pelas alterações de perfil, atribuído à incorporação de íons na matriz da membrana.

\section{CONCLUSÃO}

A partir dos resultados obtidos foi possível verificar que o sistema de envelhecimento empregado possui efeito sobre a microestrutura e estabilidade térmica das membranas. Contudo, o efeito da concentração das soluções de envelhecimento não foi um fator de impacto no processo, pois todas as membranas avaliadas apresentaram modificações estruturais análogas. Apesar disso, a caracterização microestrutural permitiu avaliar de forma distinta, como o envelhecimento ocorreu para cada membrana. A HDX 200, por exemplo, apresentou sinais de degradação e incorporação relativo ao tempo de contato, enquanto na PC 200 D, a interação membrana-solução evitou à ocorrência de hachuras em sua estrutura.

A análise térmica permitiu uma avalição mais completa de cada amostra, indicando termicamente a degradação de cada parte da membrana, o que facilitou e comprovou que o envelhecimento proporcionou alterações nos grupos funcionais e na matriz polimérica de cada membrana.

A partir desses resultados pôde-se verificar que os materiais estudados possuem capacidade de aplicação em um sistema de eletrodiálise para o tratamento e recuperação de soluções de CuHEDP. Além de mostrar que mesmo em um sistema isento de corrente elétrica as membranas já sofrem modificações cruciais em suas estruturas. Para complementar tal estudo, sugere-se a avaliação das membranas catiônicas HDX 100 e PC SK em condições similares de envelhecimento aplicado a este estudo.

\section{Agradecimentos}

Os autores agradecem ao Conselho Nacional de Desenvolvimento Científico e Tecnológico $(\mathrm{CNPq})$ e à Fundação de Apoio à Pesquisa do Estado de São Paulo (processos FAPESP n ${ }^{\circ}$ 20I 2/5 I87|-9 e no 20 I4/I335I-9). 


\section{REFERÊNCIAS}

I Strathmann H. Membrane science and technology: ion-exchange membrane separation processes. Amsterdãn: Elsevier; 2004. p. 89-I46.

2 Nikonenko WV, Kovalenko AV, Urtenov MK, Pismenskaya ND, Han J, Sistat P, et al. Desalination at overlimiting currents: State-of-the-art and perspectives. Desalination. 20I4;342:85- 106.

3 Habert AC, Borges CP, Nobrega R. Processos de separação por membranas. Rio de Janeiro: E-papers Serviços Editoriais; 2006. p. 45-80.

4 Chapotot A, Lopez V, Lindheimer A, Aouad N, Gavach C. Electrodialysis of acid solutions with metallic divalent salts: cation-exchange membranes with improved permeability to protons. Desalination. 1995; I0I(2): I4I-I53.

5 Fidaleo M, Moresi M. Electrodialysis applications in the food industry. Advances in Food and Nutrition Research. 2006;5I:265-360.

6 Cherif AT, Gavach C, Cohen T, Dagard P, Albert L. Sulfuric acid concentration with an electro-electrodialysis process. Hydrometallurgy. 1988;2I(2):191-20I.

7 Accepta. A guide to cost-effective membrane technologies for minimising wastes and effluents. Scotland: Environmental Technology Best Practice Programme; 1997. 54 p.

8 Garcia-Vasquez W, Ghalloussi R, Dammak L, Larchet C, Nikonenko V, Grande D. Structure and properties of heterogeneous and homogeneous ion-exchange membranes subjected to ageing in sodium hypochlorite. Journal of Membrane Science. 2014;452:104-II6.

9 Dammak L, Larchet C, Grande D. Ageing of ion-exchange membranes in oxidant solutions. Separation and Purification Technology. 2009;69(I):43-47.

10 Ghalloussi R, Garcia-Vasquez W, Chaabane L, Dammak L, Larchet C, Deabate SV, et al. Ageing of ion-exchange membranes in electrodialysis: a structural and physicochemical investigation. Journal of Membrane Science. 2013;436:68-78.

I I Kneifel K, Hattenbach K. Properties and long-term behavior of lon Exchange Membranes. Desalination. 1980;34(I2):77-95.

12 Robinson S, Abdullah SZ, Bérubé P, Le-Clech P. Ageing of membranes for water treatment: linking changes to performance. Journal of Membrane Science. 2016;503:177-187.

13 Scarazzato T. Tratamento de efluente contendo HEDP por eletrodiálise [dissertação]. São Paulo: Departamento de Engenharia de Materiais e Metalúrgica, Escola Politécnica, Universidade de São Paulo; 2013.

14 Vargas C. Estudo da eletrodeposição de cobre a partir de banhos alcalinos isentos de cianeto [dissertação]. São Paulo: Departamento de Engenharia de Materiais e Metalúrgica, Escola Politécnica, Universidade de São Paulo; 2008.

15 PCCell GmbH. lon exchange membranes: technical datasheet. Heusweiler; 2013.

16 Pessoa-Lopes M, Crespo JG, Velizarov S. Arsenate removal from sulphate-containing water streams by an ion-exchange membrane process. Separation and Purification Technology. 20 I6; 166:125-1 34.

17 lonashiro M. Giolito: fundamentos da termogravimetria análise térmica diferencial calorimetria exploratória diferencial. São Paulo: Giz; 2005. 96 p.

18 Kerres J, Cui W, Junginger M. Development and characterization of crosslinked ionomer membranes based upon sulfinated and sulfonated PSU crosslinked PSU blend membranes by alkylation of sulfinate groups with dihalogenoalkanes. Journal of Membrane Science. 1998; I39(2):227-24I.

19 Martos AM, Herrero M, Várez A, Levenfeld B. Synthesis and characterization of new membranes based on sulfonated polysulfone/Zn,Al-heptamolibdate LDH. Materials Letters. 2015;152:125-127.

20 Somovilla P, Villaluenga JPG, Barragán VM, Izquierdo-Gil MA. Experimental determination of the streaming potential across cation-exchange membranes with different morphologies. Journal of Membrane Science. 2016;500:16-24.

Recebido em: 2 Jul. 2019

Aceito em: 15 Fev. 2020 